Special issue of the 2nd International Conference on Computational and Experimental Science and Engineering (ICCESEN 2015)

\title{
Computational Material Analysis of Structural and Hemodynamic Model of Coronary Stent by CFD/FEA in Computer Aided Mechanical Engineering Approach
}

\begin{abstract}
Ö. KARAÇALI*
Department of Mechanical Engineering, Faculty of Engineering, Istanbul University, Avcllar, Istanbul, Turkey

With the development of new technologies, it is very popular to use a coronary stent that is a small mesh tubeshaped medical device deployed to treat narrow or weak arteries as part of a procedure called percutaneous coronary intervention. Several aspects, such as stent design, stent wire type, mechanical and material characteristics of stent have different influences on stent intervention. It has not been reported about what impacts on stent struts by the hemodynamic behavior on stent material and very few numerical studies have considered both the mechanical and hemodynamic impact of stent implementation. Computational simulation method for realization of realistic structural and hemodynamic micro environment model in this research provided valuable results of long-term functional knowledge of stent material behavior that are time consuming and expensive to determine otherwise. Computational fluid dynamics and finite element analysis simulation models were investigated and developed to evaluate engineering properties that affect stent functional attributes. These characteristics are dependences of material properties on blood flow conditions such as structural load, shear-strain rate, radial strength, and wall shear stresses, which need to be scientifically explored. To understand the material (Fe-18Cr-14Ni-2.5Mo as stainless steel 316LVM) mechanical performance of the stent, a finite element analysis simulation model was established when exposed to pulsatile blood pressure. In this study, computational fluid dynamics model was generated to calculate the wall shear stresses and strain distribution in stented vessel carrying blood to heart. The analytical analysis of mechanical and hemodynamic conduct of a stent in this investigation may help for better designs of stent, and provide deeper comprehension to support clinical cardiovascular surgeons and guide potential therapeutic strategies.
\end{abstract}

DOI: 10.12693 /APhysPolA.130.249

PACS/topics: 87.85.G-, 81.70.Bt, 82.20.Wt, 87.10.Kn, 87.15.A-

\section{Introduction}

Endovascular treatment with stenting is high medical risk, although the cardiovascular surgeons operate on the heart blood vessels to repair damage caused by stent thrombosis or coronary artery disease and may cause a heart attack or even death. Stent placement is a minimal-invasive medical procedure to treat restenosis within the coronary artery blood system. It is generally used to increase the diameter of blood vessels at narrow sections, treat artery enlargement and weakening due to abnormal blood flow behavior [1]. Coronary stent simulation is a combined problem. The blood is heterogenic, anisotropic, non-Newtonian fluid. The boundaries of the flow in the arteries are not rigid, and cannot be predicted using rigid wall, or predefined-boundarymotion calculations [2]. The methodology developed is to improve a commercially available computational fluid dynamics (CFD) method [3] to assess hemodynamic in ISO's 5840 [4]. The stress analysis by combining corresponding functions and the restenosis problem were studied by cardiovascular simulations as research concept shown in Fig. 1. The analysis of stent in this research was given in Fig. 2. Each vein is lined with a solitary layer of

*e-mail: ozdogank@istanbul.edu.tr

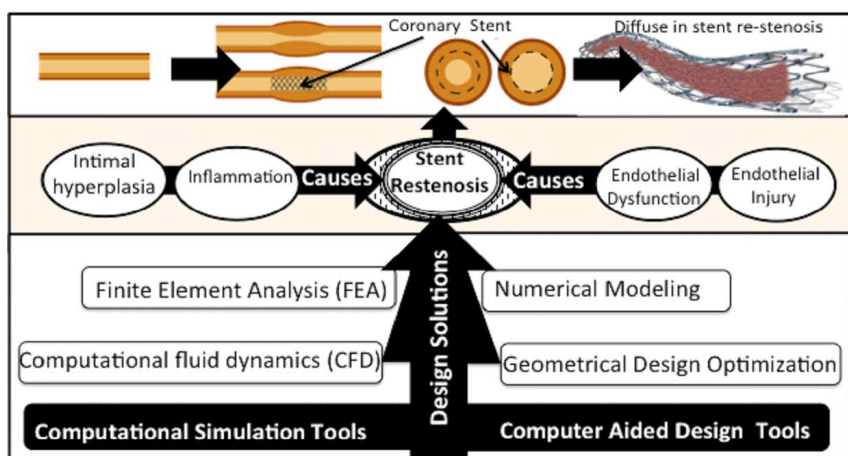

Fig. 1. Stent restenosis concept of this research.

endothelial cells that are in direct contact with the blood stream and stent.

To effectually research, model restenosis and optimize metallic stents, analyses need to be accomplished in hydrodynamic conditions that are found within vessel walls [5]. The conclusions of analysis included von Mises stress, wall shear stresses (WSS), inflow rate, and viscosity of blood. The blood fluid friction force follow up on the vessel wall varies in vascular performance around the stent [6]. The coronary stent is presented to the blood liquid at the starting phase of implantation as a consequence of liquid convection that commands the cooperation between blood and stent as shown in Fig. 2 . 


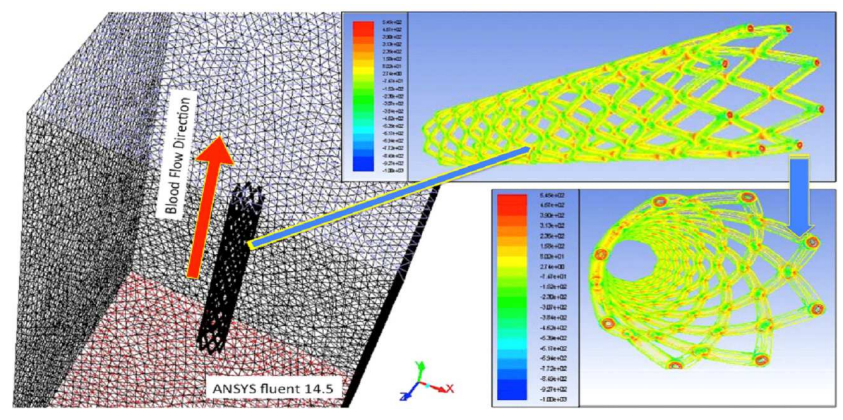

Fig. 2. Analysis of stent in investigation by CFD.

In the subsequent stage, the intima grows over the stent surface due to fluid diffusion in the interaction between tissue and stent [7]. CFD was used to construct local WSS with cardiovascular remodeling of stent. To evaluate the effects of key attributes of stent, this paper focuses on the systematic analysis of the micro hemodynamic and structural model of a coronary stent by three-dimensional CFD and finite element analysis (FEA) that can alter geometry and material properties of the strut strain.

\section{Material and methods for stent analysis}

The purpose of this study was to model a percutaneous stent and subject it to computational flute dynamic and FEA in relation to stent simulation within vascular structures. This research included two parts. First part aimed to provide CFD model, which was stimulated to examine virtual results such as WSS and blood flood. Second part included the investigation of material properties based on the local strain of a strut and mechanical stress on the stent by FEA. The stent material $\mathrm{Fe}-18 \mathrm{Cr}-14 \mathrm{Ni}-2.5 \mathrm{Mo}$ as stainless steel "316LVM" ASTM F138 ( $E=193$ GPa, $v=0.3$ ) was modeled with strut thickness of $81 \mu \mathrm{m}$ and crossing profile of $1.1-1.2 \mathrm{~mm}$. All results were generated by FEA in ANSYS. The hemodynamic loads on stent wall were assessed by unsteady fluid dynamic simulation. The flow of blood was accepted incompressible and Newtonian with viscosity of $3.71 \times 10^{-3} \mathrm{~Pa} / \mathrm{s}$ and a density of $1060 \mathrm{~kg} / \mathrm{m}^{3}$. This is an acceptable assumption in artery models [8]. Withdrawing the residual of the continuity equation and momentum imposed the convergence of blood resistance calculation set to $10^{-6}$. The blood resistance was modeled based on blood viscosity, vessel length, and vessel radius as specified by the Navier-Stokes equation as shown in Eqs. (1) and (2) below by applying the Newton-Raphson method [9] using FEA as follows:

$$
\rho \frac{\partial u}{\partial t}+\rho(u \nabla u)=\nabla\left[-p I+\left(\nabla u+(\nabla u)^{T}\right)\right]
$$
and

$$
\nabla u=0,
$$

where $u$ is the dynamic viscosity of the blood density, and $\mathrm{p}$ is the pressure in Eq. (1). In equation the term of $\rho \partial u / \partial t$ boundary condition for simulation model is equal to zero. The outcomes demonstrated that the expounded
CFD display effectively anticipated sheer configuration, shape and deposition site in endovascular stent as appeared in Fig. 3a. The developed CFD model (shown in Fig. 2) was well applied to predict the risk of restenosis formation in stent-grafts and support in selection of geometry of the endovascular prosthesis to lessen possible problems after stent-graft implantation.

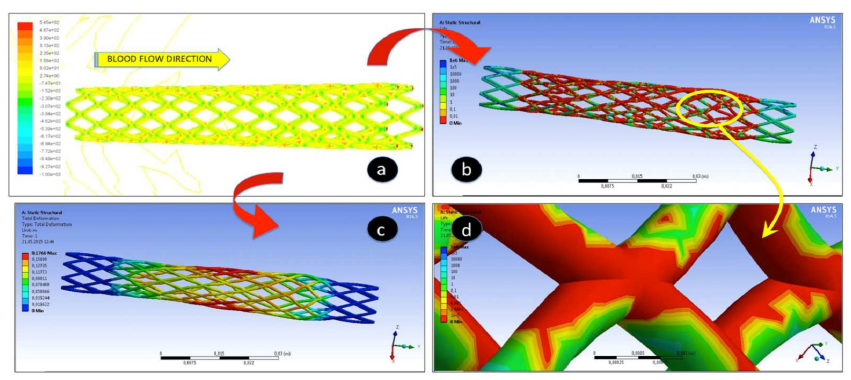

Fig. 3. Stress analysis of cardiovascular stent: (a) laminar blood flow in stent, (b) life of stent, (c) static structure of stent under laminar blood flow, (d) stress and strain effect on the stent.

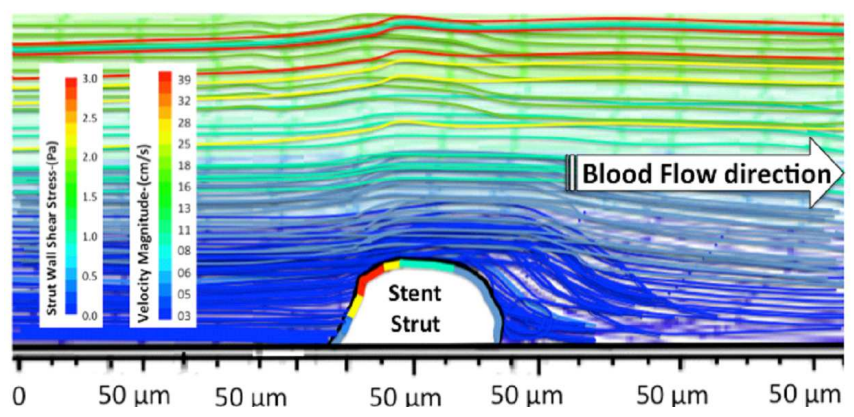

Fig. 4. Simulated blood flow around strut of stent.

All scrutinized grids were proved to be sure that results are independent of grid. Numerical calculations of laminar blood flow in the investigated domains were implemented using ANSYS FLUENT 14.5 workbench. The distribution of von Mises stresses in the stent was shown in Fig. 3b and $\mathrm{c}$ at maximum expansion instant. As can be seen in these figures, the utmost stresses and geometrical diameter changes were developed at the end of the simulation procedure as life of stent and static structure of stent under laminar blood flow. In Fig. 3a, WSS magnitude and velocity field represents for a steady state simulation. The walls of vessel and size of stent were presumed to be rigid, and a no-slip periphery state in Fig. 3d. The modeled blood density and volume as an incompressible fluid was used for simulation trials. The inlet boundary conditions were designed for steady laminar flow mode with flow rates of $2,3.2$, and $4.3 \mathrm{~mL} / \mathrm{s}$. Stent struts associated with the artery lumen generates flow turbulences near the artery barrier. These conditions involve of flow parting sections adjacent to the wall of struts generated by geometry of the strut. In Fig. 4 a computer-generated local flow disturbance area was presented to show the flow recirculation area of the strut. Flow turbulence in these areas was defined in aerody- 
namics as accelerative step flow. Adjacent wall flow disorders exerted a nominal force on the main-stream flow in the parent vessel since they were restrained close vessel walls. Consequently, struts of stent at the wall of the vessel take negligible influence on the complete flow structure within laminar blood flow. However, near-wall flow disorders produced spatial shear stress variants as principal role in stent restenosis.

Figure 5 shows a static pressure of cardiovascular stent by effect of simulated coronary laminar blood flow in artery by CFD analysis. As can be seen from Fig. 4, laminar blood flow force causes strut oscillating between 10 and $-10 \mu \mathrm{m}$ in $X$ direction while in $Y$ direction strut fluctuates between 0 and $+62 \mu \mathrm{m}$. In the simulation, percentage diameter stenosis created was corresponding to a specific stenosis due to the true functional rigorousness. In Fig. 5 pressure vs. position graph, the pressure at a given point in a medium fluctuates slightly as stent compressed by blood flow in artery.
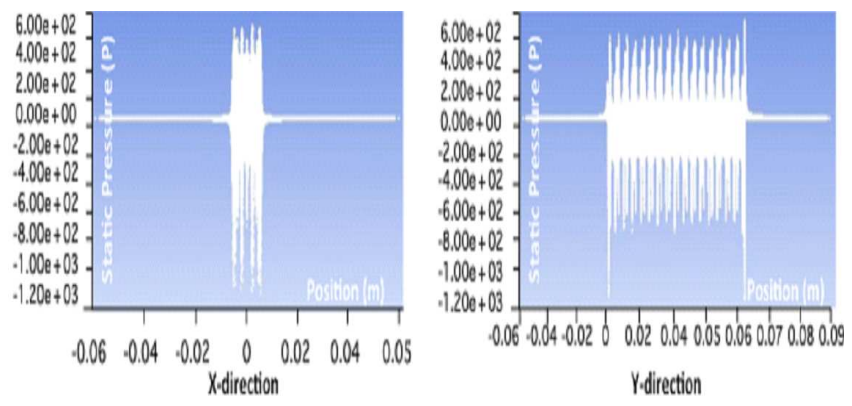

Fig. 5. Static pressure of cardiovascular stent.

All CFD and FEA evaluations were accomplished in Microsoft Windows 8 environment with Intel i7 processor $2.4 \mathrm{GHz}$ processor and $16 \mathrm{~GB}$ RAM. A populated compact mesh of tetrahedral/hexahedral layers was established to simulate near-wall effect of a coronary vein and parabolic flow profile for lumen boundary. Arterial coronary blood flow velocity were computed to plug the $3 \mathrm{D}$ remodeled lumen size with mesh entailing of 63 elements in each cross-section ranged from $0.46 \mathrm{~mm}$ to $0.81 \mathrm{~mm}$. From this research, the radial strength and the equivalent plastic strain-von Mises of stent strut thickness was observed as an important parameter for WSS in design of stent.

\section{Conclusions}

A coronary stent simulation was formed exploiting the finite element method and its mechanical behavior was evaluated with the assumptions of laminar, nonNewtonian and unsteady state flow for modeling the effect of stent on blood flow patterns, particularly artery WSS. CFD on fluid flow was employed to estimate the effect of mechanical structures in both kinematically driven models, as well as models incorporating fluid-structure interaction with hydrodynamics and stresses for coronary heart arteries. The three-dimensional CFD estimations was accomplished for pulsatile stream structure association in a disentangled coronary course, utilizing physiologically applicable laminar blood stream waveforms, with the goal of considering the impact of consistence minor deviations from the local hemodynamic developed as in Fig. 3. Development of realistic simulation models provided great benefits in this work. This research showed that the shear stress is the key biomechanical aspect calculating the vessel sizes and stent. Computergenerated models are useful to test whether the system are within these limits, or not. Thus, this model can be revised if necessary for simulation validation. As a powerful pre-operative surgical planning tool, this method may approve appropriateness of present stents and support engineers to design novel stents.

\section{Acknowledgments}

This research was financially sponsored by Istanbul University, BAP project no: 44229, Turkey.

\section{References}

[1] Ö. Karaçalı, Acta Phys. Pol. A 127, 1167 (2015).

[2] S. Pasta, A. Rinaudo, A. Luca, J. Biomech. 46, 1729 (2013).

[3] D. Perrin, J. Biomech. 48, 1868 (2015).

[4] M. Gessat, IEEE Trans. Biomed. Eng. 61, 456 (2014).

[5] A. Mehdizadeh, M.S.M. Ali, K. Takahata, S. AlSarawi, D. Abbott, J. Micromech. Microeng. 23, 1 (2013).

[6] D. Perrin, N. Demanget, Int. J. Numer. Meth. Biomed. Eng. 31, 26 (2015).

[7] Z. Chen, Y. Fan, X. Deng, Z. Xu, Artif. Org. 35, 392 (2011).

[8] Hao-Ming Hsiao, Chien-Han Lin, Ying-Chih Liao, Hsien-Yeh Chen, Tzu-Wei Wang, Curr. Nanosci. 10, 205 (2014).

[9] S. Sankaran, L. Grady, C.A. Taylor, IEEE Trans. Med. Imag. 34, 2562 (2015). 